\title{
RECURRENT RANDOM WALK OF AN INFINITE PARTICLE SYSTEM
}

\author{
BY \\ FRANK SPITZER( $\left.{ }^{1}\right)$
}

\begin{abstract}
Let $p(x, y)$ be the transition function for a symmetric irreducible recurrent Markov chain on a countable set $S$. Let $\eta_{t}$ be the infinite particle system on $S$ moving according to simple exclusion interaction with the one particle motion determined by $p$. Assume that $p$ is such that any two particles moving independently on $S$ will sooner or later meet. Then it is shown that every invariant measure for $\eta_{t}$ is a convex combination of Bernoulli product measures $\mu_{a}$ on $\{0,1\}^{S}$ with density $0 \leq \alpha=\mu[\eta(x)=1] \leq 1$. Ergodic theorems are proved concerning the convergence of the system to one of the $\mu_{\alpha}$.
\end{abstract}

1. Introduction. Let $S$ be an arbitrary countable set. On $S$ we suppose given the transition function $p(x, y)$ of an irreducible Markov chain. Further $p$ is assumed symmetric, i.e. $p(x, y)=p(y, x)$. Let $X=\{0,1\}^{S}$, with the product topology. Here is an intuitive description of the random walk of a system of particles on $S$. Let $\eta \in X$ describe an initial configuration of particles, with the interpretation that $\eta(x)=1$, or 0 , according as the site $x$ in $S$ is occupied by a single particle, or vacant. Each particle now waits a random (exponentially distributed) time with mean one. At the end of this holding time the particle attempts to jump from its site (let us call it $x$ ) to the site $y$ with probability $p(x, y)$. The jump takes place if and only if the site $y$ is vacant at that instant. It has been shown by T. M. Liggett [4] that there exists a strong Markov process $\eta_{t}, t \geq 0$, with state space $X$, which corresponds to this intuitive description. Let $S(t)$ denote the semigroup of this process. It acts on probability measures $\mu$ on $X$ in the usual way, i.e. $\nu=\mu S(t)$ means that

$$
\int_{X} v(d \eta) f(\eta)=\int_{X} \mu(d \eta)[S(t) f](\eta)
$$

for all continuous functions $f$ on $X$. A probability measure $\mu$ on $X$ (with the usual $\sigma$-algebra of subsets) is called an invariant or equilibrium measure if $\mu S(t)=\mu$ for all $t \geq 0$. The problem is, first of all, to characterize all the invariant measures; secondly, to obtain ergodic theorems. This means describing, for each invariant measure $\mu$, the class of probability measures $\nu$ on $X$ such that $\nu S(t) \Rightarrow \mu$, as $t \rightarrow+\infty$. By $\Rightarrow$ we mean weak convergence, equivalently, since $X$ is compact, convergence of the finite dimensional distributions.

Received by the editors February 26, 1973.

AMS (MOS) subject classifications (1970). Primary 60K35; Secondary 47A35.

Key words and phrases. Infinite particle systems, invariant measures, random walk, ergodic theorems.

(1) Research supported by the Mittag-Leffler Institute, Djursholm, Sweden. 
When $S$ is a finite set these problems are easy and were solved in [8]. (In this case it suffices that $p(x, y)$ be doubly stochastic rather than symmetric.) Therefore we assume that $S$ is infinite in the sequel. When $p(x, y)$ is transient the above problems were completely solved by Liggett [5]. Let $\square$ denote the compact convex set of invariant probability measures. Liggett showed that the extreme points of $\supset$ are in a one-to-one correspondence with the class $\mathcal{H}$ of functions $f$ on $S$ such that $0 \leq f \leq 1$, and

$$
\sum_{y \in S} p(x, y) f(y)=f(x), \quad x \in S .
$$

In particular, when $p(x, y)$ is such that $\&$ consists only of the constants $\alpha$, $0 \leq \alpha \leq 1, \beth$ is the well-known class $\mathcal{P}$ of symmetric probability measures on $S$, whose extreme points are the product measures $\mu_{\alpha}$, with density $\alpha, 0 \leq \alpha \leq 1$, i.e. $\mu_{\alpha}\left[\eta: \eta\left(x_{i}\right)=1, i=1,2, \ldots n\right]=\alpha^{n}$, when $x_{1}, x_{2}, \ldots, x_{n}$ are $n$ distinct sites of $S$. Liggett conjectured that this state of affairs persists when $p(x, y)$ is recurrent. This will be proved in Theorem 2 below, under the following additional condition.

(C) Let two Markov chains move according to $p(x, y)$ in the following way: at each unit time one of them is selected at random and makes a transition according to $p(\cdot, \cdot)$. Then they will sooner or later occupy the same point of $S$, with probability one.

It is nontrivial to show that condition (C) may fail to hold. This is done in a companion paper to this one by T. M. Liggett [6]. There Liggett proves the above conjecture and related ergodic theorems in the case when $p(x, y)$ is recurrent and (C) fails. His methods are different from ours, and do not apply when (C) holds.

In Theorem 3 we give a necessary and sufficient condition for a probability measure $\mu$ on $X$ to satisfy $\mu S(t) \Rightarrow \mu_{\alpha}$. Theorem 4 contains a simpler sufficient condition, communicated to by me Liggett. Theorems 5 and 6 contain a complete account of what happens when $S$ is a countable Abelian group. The random walk defined by the group invariant transition function $p(x, y)$ may be transient or recurrent. The transient case has been settled in [5]. Theorems 5 and 6 simply state that all the results are the same in the recurrent case. Theorem 6 depends crucially on Theorem 4 . We conclude by applying the results to simple random walk on the integers.

2. The finite particle system. Just as in the study of Liggett [5], the ergodic theory of the infinite particle system $\eta_{t}, t \geq 0$, with $\Sigma \eta_{0}(x)=+\infty$, can be reduced to the special case when there are only $N$ particles. Then

$$
N=\sum_{x \in S} \eta_{0}(x)=\sum_{x \in S} \eta_{t}(x), \quad t \geq 0 .
$$

It is possible to give a contracted description of this process, by looking only at the positions of the $N$ particles. Let $S^{N}$ be the product of $N$ copies of $S, D$ the set of all $\vec{x}=\left(x_{1}, x_{2}, \ldots, x_{N}\right) \in S^{N}$ such that two or more of the coordinates $x_{i}$ are equal, and let $T_{N}=S^{N} \backslash D$. Following Liggett $[5, \S 3]$, we define the transition operator $V_{N}$ on $T_{N}$ by 
$V_{N} f(\vec{x})=\frac{1}{N}\left[\sum_{i=1}^{N} \sum_{\substack{j=1 \\ i \neq j}}^{N} p\left(x_{i}, x_{j}\right)\right] f(\vec{x})$

$$
+\frac{1}{N} \sum_{i=1}^{N} \sum_{u \neq x_{j} \text { for } j \neq i} p\left(x_{i}, u\right) f\left(x_{1}, x_{2}, \cdots, x_{i-1}, u, x_{i+1}, \cdots, x_{N}\right), \quad \vec{x} \in T_{N}
$$

Thus $V_{N}$ corresponds to the transition: select one of the $N$ particles at random and let it jump according to $p(x, y)$, but only if there is no particle at $y$. Finally, let $V_{N}^{t}, t \geq 0$, be the semigroup of transition operators on $T_{N}$ defined by

$$
V_{N}^{t}=e^{-N t} \sum_{k=0}^{\infty} \frac{(N t)^{k}}{k !} V_{N}^{k}=\exp t N\left[V_{N}-I\right]
$$

This is then the contracted description of $\eta_{t}, t \geq 0$, when $\sum \eta_{0}(x)=N$; as there are $N$ particles, the expected number of attempted jumps in time $t$ is $N t$.

Let $\mathcal{B}_{N}$ be the space of real functions $f$ on $T_{N}$ with norm

$$
\|f\|=\sup _{\vec{x} \in T_{N}}|f(\vec{x})| \leq 1,
$$

and let $\delta_{N} \subset \mathcal{B}_{N}$ be the set of symmetric functions in $\mathcal{B}_{N}$. We would like to show that $V_{N}^{t} f(\vec{x})-V_{N}^{t} f(\vec{y})$ is small for large $t$ for $\vec{x} \neq \vec{y}$ in $T_{N}$. This is not true if $f \in \mathcal{B}_{N} \backslash \delta_{N}$ : Let $S=\mathbf{Z}, N=2, p(x, y)=\frac{1}{2}$ if $|x-y|=1$, and define $f(\vec{x})$ as +1 when $x_{1}<x_{2}$, and 0 when $x_{1}>x_{2}$. Then $V_{2}^{t} f(\vec{x})=1$ for all $t \geq 0$ and all $\vec{x}$ such that $x_{1}<x_{2}$, and equal to zero for all $t \geqslant 0$ and all $\vec{x}$ such that $x_{1}>x_{2}$. Fortunately we shall need only the following result.

Theorem 1. For all $N \geq 1$, and all $\vec{x}, \vec{y} \in T_{N}$

$$
\lim _{t \rightarrow \infty} \sup _{f \in \delta_{N}}\left|V_{N}^{t} f(\vec{x})-V_{N}^{t} f(\vec{y})\right|=0 .
$$

First we observe that it suffices to prove the theorem in the case when $\vec{x}$ differs from $\vec{y}$ only in one coordinate. Hence we may assume $x_{1} \neq y_{1}, x_{k}=y_{k}$ for $k=2,3, \ldots, N$. The proof will depend on a delicate coupling of two Markov processes, each with transition function $V_{N}^{t}$. This method was inspired by $R$. Holley's use of coupling to prove ergodic theorems for somewhat similar stochastic time evolution models [2], [3].

Let $X_{t}$ and $Y_{t}$ be two independent Markov processes on $T_{N}$, each with transition semigroup $V_{N}^{\prime}, N \geq 1$. Assume the initial conditions $X_{0}=\vec{x}, Y_{0}=\vec{y}$, where $x_{k}=y_{k}$ except for $k=1$. We shall construct a "coupling" of these two processes, as follows:

(a) The coupled process is a Markov process $Z_{t}=\left(X_{t}^{\prime}, Y_{t}^{\prime}\right)$ with state space $T_{N} \times T_{N}$ and with initial condition $Z_{0}=(\vec{x}, \vec{y})$. 
(b) For each $t \geq 0, X_{t}^{\prime}$ has the same probability distribution as $X_{t}$, and $Y_{t}^{\prime}$ as $Y_{t}$.

(c) Let $A_{t}$ denote the set of coordinates of $X_{t}^{\prime}$ (i.e. if the vector $X_{t}^{\prime}$ $=\left(x_{1}, x_{2}, \ldots, x_{N}\right)$ then $A_{t}$ is the unordered set $\left\{x_{1}, x_{2}, \ldots, x_{N}\right\}$ consisting of $N$ distinct elements of $S$ ). Similarly $B_{t}$ is the set of coordinates of $Y_{t}^{\prime}$. Then there exists a positive random variable $\tau$, finite with probability one, such that $A_{t}=B_{t}$ for all $t \geq \tau$.

Using properties (a), (b), (c) the proof of Theorem 1 is easy. Let $E^{(\vec{x}, \vec{y})}$ denote expectation with respect to the above process $Z_{t}$ on $T_{N} \times T_{N}$. Then (a) and (b) imply

$$
V_{N}^{t} f(\vec{x})-V_{N}^{t} f(\vec{y})=E^{(\vec{x}, \vec{y})}\left[f\left(X_{\imath}^{\prime}\right)-f\left(Y_{\imath}^{\prime}\right)\right]
$$

for all $t \geq 0$, and $f \in \mathcal{B}_{N}$. If in addition $f \in \mathcal{S}_{N}$, then $f(\vec{x})$ depends only on the set $A=\left\{x_{1}, x_{2}, \ldots, x_{N}\right\}$. So we may write $f(\vec{x})=\tilde{f}(A)$. Using (c) one has for $f \in \delta_{N}$

$$
\begin{aligned}
\left|V_{N}^{t} f(\vec{x})-V_{N}^{t} f(\vec{y})\right| & =\left|E^{(\vec{x}, \vec{y})}\left[\tilde{f}\left(A_{t}\right)-\tilde{f}\left(B_{t}\right)\right]\right| \\
& =\left|E^{(\vec{x}, \vec{y})}\left[\tilde{f}\left(A_{t}\right)-\tilde{f}\left(B_{t}\right) ; \tau>t\right]\right| \leq 2 P^{(\vec{x}, \vec{y})}[\tau>t] .
\end{aligned}
$$

Theorem 1 follows, since $\tau<\infty$ with probability one. When $N=1$ it may be noted that the conclusion of Theorem 1 is correct also without condition (C). This follows from the ergodic theorem of Orey [7].

Returning to the construction of the coupled process $Z_{t}=\left(X_{t}^{\prime}, Y_{t}^{\prime}\right)$, think of $X_{t}^{\prime}$ and $Y_{t}^{\prime}$ as the time evolutions of two systems of $N$ particles, in two containers $S_{1}, S_{2}$, each a copy of $S$. First we couple the two containers $S_{1}, S_{2}$. Each site $x \in S_{1}$ is coupled to the site $x \in S_{2}$ so that a random exponential (mean 1) clock rings simultaneously at $x \in S_{1}$ and at $x \in S_{2}$. This is done, independently, for each $x \in S$. The law of the random time evolutions in $S_{1}$ and $S_{2}$ is this: when the random clock rings at $x$ there are three possibilities:

(i) $x$ is occupied in $S_{1}$ and in $S_{2}$. Then both particles try to move according to $p(x, y)$, and this motion is also coupled in the sense that they both try to move to the same point $y$, with probability $p(x, y)$. In each container the jump to $y$ takes place if and only if $y$ is vacant.

(ii) $x$ is occupied only in one container, say in $S_{1}$. Then the particle at $x \in S_{1}$ tries to jump to $y$, with probability $p(x, y)$. The jump takes place only if $y$ is vacant.

(iii) $x$ is vacant in $S_{1}$ and $S_{2}$. Nothing happens.

It should be clear from (i), (ii), (iii) that (a) and (b) hold, i.e. $Z_{t}$ is Markovian, and the marginal processes $X_{t}^{\prime}$ and $Y_{t}^{\prime}$ are also Markov processes with the same joint distributions as $X_{t}$ and $Y_{t}$. To verify (c), suppose that at a certain time $t$, the coordinate sets $A_{t}$ and $B_{t}$ satisfy $A_{t}=C_{t} \cup \xi_{t}, B_{t}=C_{t} \cup \eta_{t}$, where $C_{t}$ is a set of cardinality $N-1$ which is the same set in $S_{1}$ and $S_{2}$. This is the case at $t=0$. To show this is true for all later $s \geqslant t$ let $C_{t}=C, \xi_{t}=x, \eta_{t}=y, x \neq y$. Thus 
$x \in S_{1}$ and $y \in S_{2}$ are the positions of the "odd" particles. We shall now compute the infinitesimal generator of $\left(\xi_{s}, \eta_{s}\right), s \geq t$, by considering all possible changes in the interval $[t, t+\Delta]$.

$(\alpha)$ The clock rings at $x$ in time $\Delta$. This results in a jump from $x$ to $a$ in container $S_{1}$, with probability $\Delta p(x, a)$, for any $a \in S \backslash C$. Note that nothing happens in $S_{2}$ since $x$ is vacant there. Thus we have the following contribution to the generator

$$
(x, y) \rightarrow(a, y) \text { with probability } \Delta p(x, a), \quad a \in S \backslash C .
$$

( $\beta$ ) The clock rings at $y$ in time $\Delta$. Just as above we get

$$
(x, y) \rightarrow(x, b) \text { with probability } \Delta p(y, b), \quad b \in S \backslash C .
$$

$(\gamma)$ The clock rings at a point $c \in C$, in time $\Delta$. Note that $c$ is occupied in both containers. Yet nothing happens to the pair of odd particles unless there is a jump from $c$ to $x$ in $S_{2}$ or from $c$ to $y$ in $S_{1}$. In the first case the coordinates of the "odd" pair $\left(\xi_{t}, \eta_{t}\right)$ change from $(x, y)$ to $(c, y)$; in the second case from $(x, y)$ to $(x, c)$. Schematically we have

$$
\begin{array}{ll}
(x, y) \rightarrow(c, y) \text { with probability } \Delta p(c, x), & c \in C, \\
(x, y) \rightarrow(x, c) \text { with probability } \Delta p(c, y), & c \in C .
\end{array}
$$

First of all this classification shows that the coordinate sets of the two processes $X_{t}^{\prime}$ and $Y_{t}^{\prime}$ will never differ by more than one pair of points if this is true at time 0 . To verify (c) combine the specific expressions for the generator in cases $(\alpha)$, $(\beta),(\gamma)$ making use of the symmetry of $p(x, y)$ to write $p(c, x)=p(x, c), p(c, y)$ $=p(y, c)$ in part $(\gamma)$. The result is that in time $\Delta$ a change of $\left(\xi_{t}, \eta_{t}\right)=(x, y)$ to $(x, a)$ has probability $\approx \Delta p(y, a)$ for all $a \in S$ and to $(a, y)$ the probability is $\approx \Delta p(x, a)$ for all $a \in S$. But this is just the statement that the infinitesimal generator of $\left(\xi_{t}, \eta_{t}\right)$ is exactly the same as of a pair of independent Markov processes with transition semigroup $V_{1}^{t}$ (whose generator is the sum of the generators $p(x, y)-\delta(x, y)$ for each process). Since this generator is a bounded operator it clearly defines the process $\left(\xi_{t}, \eta_{t}\right)$ uniquely up to the first time $\tau$, when $\xi_{t}=\eta_{t}$. This stopping time is finite with probability one since the pair $\left(\xi_{t}, \eta_{t}\right)$ observed only at instants of change behaves just like the discrete time Markov chain in condition (C). After time $\tau$ we have identical coordinate sets in $S_{1}$ and $S_{2}$, i.e. $A_{t}=B_{t}$ for all $t \geq \tau$. This is clear from (i), (ii), (iii) above.

3. The infinite particle system. For an arbitrary probability measure $\mu$ on $X$, define the system of its correlation functions $\rho^{(N)}, N \geq 1$, by

$$
\begin{aligned}
\rho^{(N)}(\vec{x}) & =\mu\left[\eta \mid \eta\left(x_{1}\right)=\eta\left(x_{2}\right)=\cdots=\eta\left(x_{N}\right)=1\right], \\
\vec{x} & =\left(x_{1}, \ldots, x_{N}\right) \in T_{N} .
\end{aligned}
$$


Note that each $\rho^{(N)}$ is a symmetric function on $T_{N}$. If $\rho_{i}^{(N)}$ are the correlation functions of a family of measures $\mu_{t}, t \geq 0$, and if $\mu_{t}=\mu_{0} S(t), t \geq 0$, then the correlations satisfy

$$
\rho_{i}^{(N)}(\vec{x})=V_{N}^{\prime} \rho_{0}^{(N)}(\vec{x}), \quad N \geq 1, \vec{x} \in T_{N} .
$$

This basic result occurs in [5] and [8]. It is easily derived by checking that the correlations satisfy the diffusion equation

$$
\frac{\partial}{\partial t} \rho_{i}^{(N)}(\vec{x})=N\left(V_{N}-I\right) \rho_{i}^{(N)}(\vec{x}), \quad t \geq 0, \vec{x} \in T_{N} .
$$

Since the operators $V_{N}-I$ are bounded, (3.1) gives the unique solution. It is well known that a probability measure $\mu$ on $X$ is uniquely determined by its correlations. This fact together with (3.1) and Theorem 1 yields the ergodic theory for the infinite particle system $\eta_{t}, t \geq 0$.

Theorem 2. The set of all equilibrium measures is $\mathfrak{P}$.

Proof. The set $\mathscr{P}$ of symmetric measures is the closed convex hull of the product measures $\mu_{\alpha}$ on $X$. These have constant correlation functions. Hence (3.1) implies that $\mathscr{P} \subset \supset$. Conversely suppose $\mu \in \beth$ with correlations $\rho^{(N)}$. Then if $\rho_{t}^{(N)}$ are the correlations of $\mu S(t)$, we have $\rho_{t}^{(N)}=\rho^{(N)}$. Theorem 1 applied to (3.1) gives

$$
\begin{aligned}
\left|\rho^{(N)}(\vec{x})-\rho^{(N)}(\vec{y})\right| & =\left|V_{N}^{t} \rho^{(N)}(\vec{x})-V_{N}^{t} \rho^{(N)}(\vec{y})\right| \\
& \leq \sup _{f \in \delta_{N}}\left|V_{N}^{t} f(\vec{x})-V_{N}^{t} f(\vec{y})\right| \rightarrow 0, \text { as } t \rightarrow \infty .
\end{aligned}
$$

Hence each $\rho^{(N)}$ is constant on $T_{N}$. Therefore the cylinder set probabilities of $\mu$ (and hence $\mu$ itself) are invariant under all finite permutations of $S$. This property characterizes $\mathcal{P}$ by de Finetti's theorem [1].

Theorem 3. Let $\mu_{\alpha}$ be the product measure on $X$ with density $\alpha, 0 \leq \alpha \leq 1$. Then $\mu S(t) \Rightarrow \mu_{\alpha}$ as $t \rightarrow \infty$ if and only if the correlations of $\mu$ satisfy

(a) $\lim _{t \rightarrow \infty} V_{1}^{t} \rho^{(1)}(x)=\alpha, x \in S=T_{1}$;

(b) $\lim _{t \rightarrow \infty} V_{2}^{t} \rho^{(2)}(\vec{x})=\alpha^{2}, \vec{x} \in T_{2}$.

Proof. If $\mu S(t) \Rightarrow \mu_{\alpha}$ then $\rho_{t}^{N}(\vec{x}) \rightarrow \alpha^{N}$ as $t \rightarrow \infty$ for each $N \geq 1$. Thus (3.1) implies the necessity of (a), (b). Suppose now $\mu$ is such that (a) and (b) hold. Since $S$ is countable we may find a sequence $t_{n} \lambda+\infty$ such that

$$
\lim _{n \rightarrow \infty} V_{N}^{l_{n}} \rho^{(N)}(\vec{x})=c_{N}(\vec{x})
$$

exists for each $N \geq 1$, and all $\vec{x} \in T_{N}$. It follows from Theorem 1 that each $c_{N}$ is constant on $T_{N}$. Now the $c_{N}$ are limits of correlation functions of a sequence of probability measures. By compactness of the set of probability measures on $X$, we know that $\left\{c_{N}\right\}$ is the sequence of correlations of a probability measure $\nu$ on 
$X$. As mentioned in the proof of Theorem 2, that measure is in $\mathcal{P}$. Hence it is of the form

$$
\nu=\int_{0}^{1} \mu_{\gamma} d F(\gamma)
$$

Thus

$$
c_{N}=\int_{0}^{1} \gamma^{N} d F(\gamma), \quad N \geq 1,
$$

for some probability distribution $F$ on $[0,1]$. But by hypotheses (a) and (b)

$$
c_{1}=\int_{0}^{1} \gamma d F(\gamma)=\alpha, \quad c_{2}=\int_{0}^{1} \gamma^{2} d F(\gamma)=\alpha^{2} .
$$

This implies that $F$ concentrates its mass at $\gamma=\alpha$. Hence $c_{N}=\alpha^{N}$ for all $N \geq 1$. But we have proved this independently of how the subsequence $t_{n}$ in (3.3) was chosen. Therefore

$$
\lim _{t \rightarrow \infty} V_{N}^{t} \rho^{(N)}(\vec{x})=\alpha^{N}, \quad N \geq 1, \vec{x} \in T_{N} .
$$

This implies that $\mu S(t) \Rightarrow \mu_{\alpha}$.

Condition (b) in Theorem 3 is difficult to verify in practice, because of the interaction of the two-particle system moving according to $V_{2}^{t}$. It would be simpler to deal with the independent two-particle system represented by the operator $U_{2}^{t}=\exp 2 t\left(U_{2}-I\right)$, where

$$
U_{2} f(\vec{x})=\frac{1}{2} \sum_{u}\left[p\left(x_{1}, u\right) f\left(u, x_{2}\right)+p\left(x_{2}, u\right) f\left(x_{1}, u\right)\right], \quad \vec{x} \in S^{2} .
$$

The following result is due to $T$. Liggett.

Theorem 4. The conditions in Theorem 3 remain sufficient for $\mu S(t) \Rightarrow \mu_{\alpha}$, if $V_{2}^{t}$ in condition (b) is replaced by $U_{2}^{t}$.

Proof. Suppose that the modified condition (b) holds. Observe that

$$
\rho^{(2)}(\vec{x})=\mu\left[\eta: \eta\left(x_{1}\right)=\eta\left(x_{2}\right)=1\right], \quad \vec{x}=\left(x_{1}, x_{2}\right) \in S^{2}
$$

is a bounded, symmetric, positive definite function on $S^{2}$. Liggett has shown in Lemma 2.7 of [6] that every such function $f$ satisfies

$$
V_{2}^{t} f(\vec{x}) \leq U_{2}^{t} f(\vec{x}), \quad \vec{x} \in T_{2} .
$$

Therefore

$$
\limsup _{t \rightarrow \infty} V_{2}^{t} \rho^{(2)}(\vec{x}) \leq \alpha^{2}, \quad \vec{x} \in T_{2} .
$$

Suppose now that (a) holds, and that for some subsequence $t^{\prime} \rightarrow \infty$, we obtain a limit less than $\alpha^{2}$. Just as in the proof of Theorem 3 this would imply 


$$
\int_{0}^{1} \gamma^{2} d F(\gamma)<\left[\int_{0}^{1} \gamma d F(\gamma)\right]^{2}=\alpha^{2},
$$

which is impossible. Hence (b) in Theorem 3 holds, which proves Theorem 4.

Remark. Theorem 4 may be expressed, as in Theorem 1.5 of [5], by saying that $\mu S(t) \Rightarrow \mu_{\alpha}$, provided

$$
\lim _{t \rightarrow \infty} e^{-t} \sum_{n=0}^{\infty} \frac{t^{n}}{n !} \sum_{y \in S} p^{(n)}(x, y) \eta_{0}(y)=\alpha
$$

in probability, with respect to $\mu$, for each $x \in S$. Since $p(x, y)$ is recurrent it even suffices, by Orey's ergodic theorem [7], to assume that (3.4) holds for a single point $x \in S$.

Further simplification results if we assume that the probability measure $\mu$ is concentrated at a point of $X$, i.e. that the initial configuration $\eta_{0}(x), x \in S$, is a given nonrandom assignment of zeros and ones. In this case (3.4) is evidently equivalent to condition (a) in Theorem 3. Therefore (3.4) is necessary and sufficient for $\mu S(t) \Rightarrow \mu_{\alpha}$, whenever $\mu$ is concentrated on the single point $\eta_{0} \in X$.

4. Random walk on a group. Suppose now that $S$ is an additive Abelian group, and that $p(x, y)=p(0, y-x)$ for all $x, y \in S$. In this case condition (C) holds automatically when $p(x, y)$ is recurrent. To show this, let $X_{t}, Y_{t}$ be two independent random walks on $S$, both starting at 0 , i.e. processes with transition semigroup $V_{1}^{t}, t \geq 0$. Then $X_{t}-Y_{t}$ is exactly the same process as $X_{2 t}$. Hence $X_{t}-Y_{t}=0$ infinitely often with probability one. Therefore $\left(X_{t}, Y_{t}\right)$ visits the diagonal $D \subset S^{2}$ with probability one from $(0,0)$. As $p(x, y)$ is irreducible the same is true for any starting point. Therefore condition (C) holds. Thus Theorem 2 holds. Since Liggett ([5]) has proved the corresponding fact in the transient case we have

Theorem 5. In the group invariant case, when $p$ is symmetric and irreducible, the set of equilibrium measures is always $\mathfrak{P}$.

In the transient case Liggett has proved [5] that $\mu S(t) \Rightarrow \mu_{\alpha}$ for every ergodic measure $\mu$ with density $\alpha$, which is invariant under translation by arbitrary elements of the group $S$. This is also true in the recurrent case.

Theorem 6. Suppose that $\mu$ is a translation invariant ergodic probability measure on $X$, with density $\mu[\eta: \eta(x)=1]=\alpha$. Let $p(\cdot, \cdot)$ be symmetric, irreducible, group invariant, and either recurrent or transient. Then $\mu S(t) \Rightarrow \mu_{\alpha}$.

Proof. The proof is exactly that given by Liggett in the transient case; see Theorem 5.6 in [5]. It consists in verifying the conditions in Theorem 4 above and is independent of whether the random walk is recurrent or transient.

Example. Let $S=\mathbf{Z}$, the integers, and $p(x, y)=\frac{1}{2}$ if $|x-y|=1$, and 0 otherwise. Then the equilibrium measure $\mu_{\alpha}$ is approached by the time evolution if the initial sequence $\eta_{0}(x), x \in Z$, is any stationary 0,1 valued ergodic process with mean $\alpha$. Suppose now that $\eta_{0}(x), x \in \mathbf{Z}$, is a given nonrandom sequence of 
zeros and ones. By the remark following the proof of Theorem 4 we get convergence to $\mu_{\alpha}$ if and only if

$$
e^{-t} \sum_{n=0}^{\infty} \frac{t^{n}}{n !} \sum_{k \in \mathbf{Z}} p^{(n)}(0, k) \eta_{0}(k)=\sum_{k=-\infty}^{\infty} e^{-t} I_{|k|}(t) \eta_{0}(k)
$$

tends to $\alpha$ as $t$ tends to infinity. Here $I_{k}$ denotes a familiar Bessel function. By direct computation, or by use of a local central limit theorem, this can be proved for every initial configuration with density $\alpha$, i.e. satisfying

$$
\lim _{n \rightarrow \infty} \frac{1}{2 n+1} \sum_{k=-n}^{n} \eta_{0}(k)=\alpha .
$$

\section{BiBLIOGRAPHY}

1. E. Hewitt and L. J. Savage, Symmetric measures on Cartesian products, Trans. Amer. Math. Soc. 80 (1955), 470-501. MR 17, \#863.

2. R. Holley, An ergodic theorem for interacting systems with attractive interactions, Z. Wahrscheinlichkeitstheorie und Verw. Gebiete 24 (1972), 325-334.

3. , Recent results on the stochastic Ising model, Rocky Mountain Math. J. (to appear).

4. T. M. Liggett, Existence theorems for infinite particle systems, Trans. Amer. Math. Soc. 165 (1972), 471-481.

5. A characterization of the invariant measures for an infinite particle system with interactions, Trans. Amer. Math. Soc. 179 (1973), 433-453.

6. A characterization of the invariant measures for an infinite particle system with interactions. II, Trans. Amer. Math. Soc. 198 (1974), 201-213.

7. S. Orey, An ergodic theorem for Markov chains, Z. Wahrscheinlichkeitstheorie und Verw. Gebiete 1 (1962), 174-176. MR 26 \#3117.

8. F. Spitzer, Interaction of Markov processes, Advances in Math. 5 (1970), 246-290. MR 42 \#3856.

Department of Mathematics, Cornell University, Ithaca, New York 14850 